\title{
A Review Paper on MIMO Based OFDMA System in Digital Communications System
}

\author{
Mohammad Yusuf \\ BTKIT, Dwarahat
}

\begin{abstract}
In communication system, the combination of MIMO and OFDMA system is the most promising technology for high bit rate provided for the next generation wireless communication network. This paper presents a review on the structure of MIMO antenna; In MIMO system more than one antenna is used for communication by which capacity and ranged of the system is increased. In MIMO system antennas are used in both transmitter and receiver to improve the communication performance, whereas the OFDMA is the multicarrier multiple access method. MIMO-OFDMA is commonly used for communication system due to its high transmission rates and robustness against multipath fading
\end{abstract}

Keywords: MIMO, Wireless Communications, Channel Coding, OFDMA

\section{INTRODUCTION}

Recently, there is high demand for multimedia for services in wireless communication system requires high transmission rates. Because of which fading and Intersymbol interference will occur in the system. OFDMA is commonly used in WLANs. OFDMA allowing subchannels in the frequency domain and increases the transmission rates . MIMO-OFDMA system makes demand for the most versatile and efficient solution for high spectral efficiency requirements. MIMO-OFDMA, when OFDM signal is transmitted through a number of antennas to achieve diversity(High Transmission Rates), then it is known as MIMO-OFDMA. MIMO system can be implemented by several types, for combat fading we need to send the same signals through various MIMO antenna and at receiving end all the signals received by MIMO antennas will receive the same signals travelled through various paths. MIMO systems provide a gives of benefits over single-antenna-to-single-antenna transmission. Vulnerable to fading is diminished by the spatial diversity proposed by multiple spatial route. Under many environmental states, the power needs attached with extremely higher spectral efficiency transmission may be frequently diminished by not considering the contracted areas of the information-theoretic capability bound. Here, efficiency of spectrum is given as the total number of data bits per second per Hertz transferred from one array to array. Capacity development and bit error rates (BERs) has expanded the recent interest in multiple-antenna systems. With the benefits, nevertheless, comes a valuation in hardware difficulties. Radio front end has problem, size and valuation that scale along total number of antennas. It is feasible to control the cost and simultaneously capture several benefits of MIMO structure by a method known as selecting the antenna. A simple presentation of the MIMO systems is given in Fig. 1.

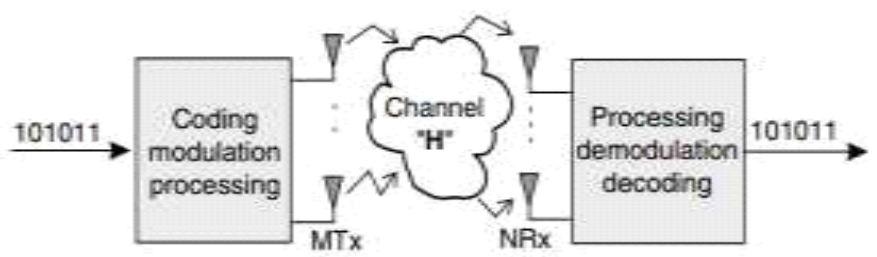

Fig.1 Schematic Representation of a General MIMO Wireless scheme

MIMO antenna systems uses a special technique known as Spatial Multiplexing in which the transmission and receiving of data takes place in matrix form not in any vector channel which allows MIMO systems to transmit more than one parallel signal synchronously in the same frequency band increasing the spectral efficiency. The data block is coded with the vector encoder and transferred synchronously by M transmitters. MIMO channel of radio precede disturb to the signal. The receiver have $\mathrm{N}$ numbers of antennas. Every antenna takes signals from every $\mathrm{M}$ transferred antennas, and therefore accepted signals display inter-channel involvement. Accepted signals snidely changed to base band, Observed once each symbol of that interval. Processed MIMO units evaluate transferred data blocks along sampled base-band signals. Vector decoder convert parallel-to-serial, then this merges input data parallel blocks to single output data block Regarded as development transmission System that is wireless design, multiple antenna systems fuel the ever growth of data rate requirements of advanced technologies such as Long-Term Evolution, Wireless-LAN etc. The use of MIMO systems in wireless communications have led us to overcome several problems related to wireless communication like fading, multipath, etc. The concept of spatial variety used for huge amount of reliability of data and spatial multiplexing is used to 


\title{
International Advanced Research Journal in Science, Engineering and Technology
}

\author{
Vol. 5, Issue 11, November 2018
}

expand rates of data for transmission channels. Mainly original route from sender to recipient and many of the transferred signals take alternate route to the recipient like off objects bouncing, surface of atmosphere. Signals transverse indirect path arrives at the receiver with a delay and often gets effected. A common programme associated with not stronger multirooted signals is to avoid them but power in this case is discarded. robust multipath signals are strongly avoided and also can minimize the exhibition of the wireless device. The robust signal in each instant in time is received and enumerate variant in received signal that are late to enforce the top of calibration this concept is used in MIMO and thus MIMO takes advantage of multipath. Multipath arise when the mismatched signals arrive at the receiver at various times. In place of expanded bit rate, MIMO can also be used to utilize heterogeneity. Transmit heterogeneity approaches are available from Wireless CDMA release 99 and will also form part of Long Term Evolution as one MIMO mode. In case the channel states are not permissible in spatial multiplexing, a transmit heterogeneity approaches will be used instead, so switching between these two MIMO modes is suitable based on channel states. Transferred heterogeneity is used when the chosen number of block is one. Similar encoded data and transferred via several antennas which adequately double effect in the channel. This enhance the SNR for cell edge performance. In MIMO systems the trading-off occur among spatial multiplexing and the spatial heterogeneity. Refined spatial multiplexing permissible for completely autonomous antennas uses. In spite of, it provides restricted heterogeneity gain and a minimal approach for appropriate BER (Bit Error Rate) aim. The chance of a linear capability extension with the antenna numbers is splendid, particularly known as that the increased power, encoding the characters along the stream may result in extra coding and heterogeneity gain, that may be provide increased performance and robustness, although the bit rate is kept at the same state. It is also feasible to drop few bit rate for more heterogeneity.

\section{ADAPTIVE MIMO SYSTEMS}

In Adaptive Coding Modulation, if the channel information is present at the transmitting end of the system then telecom structure may alter total rate with the present states of the channel. ACM when used with the MIMO antenna systems can be used for increasing the selection mode. Generally the systems that uses the MIMO systems with ACM are more dependable and long-lasting. The information at the receiving channel is more easily known than the information at the transmitting channel in which the medium may be calculated by the instructing order. For the feedback data from receiving end to transmitting end an equipment is required except for the reciprocal channel in which same channel or information is used in both the transmitting and receiving ends like in duplex systems. Various schemes were proposed but beam forming has been selected for the adaptation. MIMO antenna systems has CSI which is significant for selecting one from the previously defined modes of actions. SNR is used in spatial multiplexing scheme for selecting spatially multiplexed blocks. The selection of transmitting antenna is based on selecting a set of transmitting antenna to meet certain requirements.

\section{OFDM AND MIMO}

OFDM is also known as Orthogonal frequency division multiplexing. In this the digital data is encoded on frequencies with multiple carrier. Here orthogonal signals are carried in frequency. The mechanism used here is for $4 \mathrm{G}$ systems. The microwave access is used as an $3.75 \mathrm{G}$ version which is WiMAX. OFDM which is a key wireless broadband technology. Here, by broadband it means that a wireless system which has a large bandwidth which means that OFDM is a broadband technology which operates on a huge bandwidth or a very large band for example the bandwidth of OFDM is $20 \mathrm{MHz}$ which is much higher when it is compared with the GSM. Since it has a large bandwidth therefore naturally the data rates are higher and the higher data rates are used in 3G \& 4G wireless systems. These higher data rates are of the order of hundreds of Mbps. Considering a communication system has bandwidth (B) and a single carrier of certain frequency. Now for instance if $\mathrm{B}=10 \mathrm{MHz}$. So, this is a broadband system of bandwidth $10 \mathrm{MHz}$. The symbol time is given by below expression:

$$
\begin{gathered}
\mathrm{T}=\frac{1}{\mathrm{~B}} \\
\mathrm{~T}=\frac{1}{10 * 10^{6}} \\
\mathrm{~T}=0.1 \mu \mathrm{S}
\end{gathered}
$$

The delay spread (Td) of a wireless system is approximately $2-3 \mu$ s. Therefore, symbol time is much less than delay spread. Here, delay spread is 20 times that of symbol time. The problem of ISI is generally arised as the delay spread is larger then the symbol time. So, symbol time (T) has inverse relation with bandwidth (B) means more is the bandwidth of the channel, lesser is the symbol time and if this $\mathrm{T}$ is less than Td this leads to a problem of ISI. Due to the interruption there is massive loss in the capability for communication system. Therefore ISI leads to degradation in performance and this is a limitation of broadband system. To solve this problem instead of taking the entire bandwidth (B) it is split into sub bands (smaller bands). Earlier, there was single carrier and now there are multiple carriers in every sub band which is nothing but subcarriers. If it is assumed there is an split of $\mathrm{N}$ sub bands which is mentioned below: $\mathrm{Bs}=\mathrm{B} / \mathrm{N}$ 


\section{International Advanced Research Journal in Science, Engineering and Technology}

Vol. 5, Issue 11, November 2018

For example: If $\mathrm{B}=10 \mathrm{MHz}$ and $\mathrm{N}=1000$ then bandwidth for particular sub band is,

$$
\mathrm{B}_{\mathrm{S}}=\frac{10 \mathrm{MHz}}{1000}=10000=10 \mathrm{kHz}
$$

Also, each sub band has symbol time (Ts) is given by,

$$
\begin{aligned}
& \mathrm{T}_{\mathrm{s}}=\frac{1}{\mathrm{~B} / \mathrm{N}}=\frac{\mathrm{N}}{\mathrm{B}} \\
& \mathrm{T}_{\mathrm{s}}=\frac{1}{10 \mathrm{kHz}}=100 \mu \mathrm{s}
\end{aligned}
$$

Now, if this symbol time of each sub-band (Ts) is compared with delay spread (Td) then Ts is much greater than Td. Therefore, there is no Inter Symbol Interference (ISI) in this system which has multiple sub bands and subcarrier in each band and such a system is called as Multi Carrier Modulated (MCM) system. This system is the basis of OFDM system. Application of OFDM modulation with in MIMO-architected approach make a powerful system that has the capability to completely dismiss fading and fulfils the need for high throughput. Extanded capability of MIMO channels can converted into maximum throughput endowed that reasonable coding is done major to transmission.

\section{ADVANTAGES OF MIMO SYSTEMS}

There are various advantages of MIMO systems antenna which are array obtain, intervention reduction, and variety gain. MIMO systems antennas has been used for transmitting or receiving multiple benefits of antenna and that break the tradition of old various antenna systems by multiplexing gain of the various antennas. As it is difficult to tell about the topmost diversity with topmost multiplexing both then agreement is made. MIMO adaptive systems can alter the multiple antennas to the present conditions and at the same time can be used for increasing the yield and accuracy.

\section{FUTURE SCOPES}

In this paper of wireless communication, we have properly described OFDM and OFDMA and MIMO system to be introduced related modulation scheme OFDM and access scheme OFDMA, MIMO-OFDMA method to improve the diversity as well as capacity in cellular system. Based on literature study in this paper have used that OFDMA is much better suited to a multiple channel then the usual signal carrier translation method such as 16-QAM requirement or desire for high transmission rate for wireless communication has been increasing drastically throughout the last decade. study of various interference effect in a MIMO-OFDMA system in this review paper most of them use Estimation and compensation process has an efficient algorithm has to be formed which takes into account all the interferences and It is a part of our future Work.

\section{CONCLUSION}

MIMO has been a greatest achievement when we talk about wireless communication. The presentation and the ability is expanded significantly. As there is an evolutionary growth the various standards are defined. The previous versions were not so compatible so there were the need of new versions as WiMAX. Traditional methods can't solve the limitations. the desire for high data rates wireless communication have been increasing drastically throughout the last decade. This paper has looked into the role of MIMO- OFDMA within the wireless communication and it is advantages over single provider transmission. There area also a few limitations of this technique which is often removed with a guide of suitable techniques.

\section{REFERENCES}

[1]. Gesbert, D.; Shafi, M.; Da-shan Shiu, Smith, P.J.; Naguib. A. From theory to practice: an overview of MIMO spacetime coded wireless systems. IEEE J. Sel. Areas Commun. 2003, 21, 281-302

[2]. Gershman, A.B.; Sidiropoulus, N.D. Space-Time Processing for MIMO Communications; John Wiley \& Sons Inc.: 2005.

[3]. Foschini, G.J.; Gans, M.J. Layered space-time architecture for wireless communication in a fading environment when using multiple antennas. Bell Labs Syst. Tech. J. 1996, 1, 41-59.

[4]. Allen, B.; Ghavami, M. Adaptive Array Systems: Fundamentals and Applications; John Wiley \& Sons Inc.: 2005. 8. Vucetic, B.; Yuan, J. Space-Time Coding; Wiley \& Sons Inc.; 2003.

[5]. Paulraj, A.J.; Gore, D.A.; Nabar, R.U.; Bo“lcskei, H. An overview of MIMO communications—a key to gigabit wireless. Proc. IEEE. 2004,92 , $198-218$.

[6]. Kavita Devi, Rajneesh Thakur; "Performance Enhancement of MIMO-OFDMA: A Review Paper"; International Journal of Computer Science and Communication Engineering, Volume: 2 Issue: 2 (May 2013).

[7]. D.L kumari,"A Review Paper on Performance Analysis of MIMO based OFDMA System under fading channel: International Journal of Electronics \& Communication Engineering and Technology (IJECET) June 2017 